\title{
¿Cómo impactará la tecnología al Derecho Comercial?*
}

\section{Alejandro Rafael Morales Cáceres}

Abogado por la Universidad de Lima. Miembro Asociado de ADV Editores - Revista ADVOCATUS.

\author{
SUMARIO: \\ I. Introducción. \\ II. ¿Por qué es necesario regular la tecnología? \\ III. Inteligencia Artificial. \\ IV. Blockchain. \\ V. Criptomonedas \\ VI: Conclusión.
}

* El gran motor del cambio: La tecnología" - Alvin Toffler 


\title{
RESUMEN:
}

En un mundo en donde la tecnología avanza de forma acelerada y los negocios comienzan a implementarla, los juristas debemos preguntarnos cuál es su grado de impacto sobre el Derecho Comercial, y, si resulta necesario crear leyes que la promuevan o que la restrinjan. Asimismo, cabe cuestionarse si estas nuevas tecnologías impactarán las estructuras societarias que actualmente conocemos.

Palabras Clave: Derecho Comercial, Tecnología, Inteligencia Artificial, Criptomonedas, Blockchain.

\begin{abstract}
:
In a world where technology is advancing at an accelerated pace and businesses are beginning to implement it, we jurists must ask ourselves how this impacts Commercial Law and if it is necessary to create laws that promote or restrict its use. Also, it is questionable whether these new technologies will impact the societal structures that we currently know.
\end{abstract}

Keywords: Commercial Law, Technology, Artificial Intelligence, Cryptocurrencies, Blockchain.

\section{INTRODUCCIÓN}

En el año 2014, los medios de comunicación dieron a conocer la noticia que Deep Knowledge Ventures, una firma dedicada al venture capital con sede en Hong Kong, había designado como el sexto miembro de su Directorio a"Vital", una "Inteligencia Artificial" —en adelante, "Al" — capaz de predecir tendencias del mercado invisibles para el entendimiento humano. En otras palabras, se nombró como "director" a un algoritmo capaz de anticipar lo que va a suceder en materia de comercio, con mucha mayor antelación de lo que lo harían expertos humanos.

"Vital" es el acrónimo en inglés de Validating Investment Tool for Advancing Life Sciences Herramienta de Validación de Inversiones para Ciencias Vivas Avanzadas - y se encarga de analizar diferentes bases de datos de empresas de investigación en el sector médico, que comparten su información con el fin de predecir inversiones exitosas. Por ejemplo, "Vital" ayudó a aprobar dos decisiones de inversión, concretamente para financiar Insilico Medicine, una compañía que desarrolla métodos asistidos por computadora para el descubrimiento de fármacos en la investigación del envejecimiento, y Pathway Pharmaceuticals, empresa que selecciona y clasifica terapias personalizadas contra el cáncer, sobre la base de una plataforma tecnológica.

A pesar que, en términos jurídicos, "Vital" no es considerado como un director, conforme a las leyes corporativas de Hong Kong, si ha sido reconocido como la primera inteligencia artificial que ocupa el cargo de director de una sociedad en el mundo. El cuasi nombramiento de "Vital" como parte del Directorio, marca un hito fundamental para el Derecho Societario, ya que demuestra el impacto de la inteligencia artificial en la toma de decisiones corporativas. A medida que las industrias se sofistiquen y las decisiones comerciales deban ser tomadas sobre la base de numerosos y complejos conjuntos de datos, la Inteligencia Artificial, que además tiene la capacidad de aprender, será usada con mayor frecuencia en el futuro. En otros términos, el desarrollo tecnológico conducirá inevitablemente al apoyo y, posiblemente, a la sustitución de directores corporativos por algoritmos como "Vital". Se estima que, dentro de diez a quince años, los sistemas de Inteligencia Artificial podrán tomar las decisiones en una corporación, sin apoyo humano.

Así como en el caso de la Inteligencia Artificial, observamos que distintas tecnologías como el "Blockchain" o las criptomonedas podrían cambiar las estructuras y los procesos dentro de la corporación y, por consiguiente, las normas del Derecho Societario deberán adecuarse a esta nueva realidad. Thomas Hobbes sostuvo en su libro "Leviathan" que, si los hombres permanecieran en estado natural, es decir, sin leyes ni gobiernos, terminarían solos, pobres y embrutecidos a causa de las luchas que inevitablemente se desatarían entre ellos. En forma análoga, si la tecnología se desarrollase en estado natural, es 
decir, sin ningún tipo de regulación, el sistema corporativo terminaría destruido.

\section{II. ¿POR QUÉ REGULAR LA TECNOLOGÍA?}

Antes de iniciar un análisis de cómo estas nuevas tecnologías pueden afectar al Derecho Comercial, considero necesario el preguntarnos si es preciso regularlas, vía la creación de leyes o la modificación de aquellas ya existentes. La innovación y la creatividad siempre han conllevado a que se creen máquinas o programas que mejoran la calidad de vida; sin embargo, la regulación puede generar mayores costos en el proceso de creación y, por tanto, menos tecnología que mejore la eficiencia en los procesos. En otras palabras, la reglamentación puede evitar que se frenen emprendimientos que coadyuven al ser humano en su día a día.

Si bien hay opiniones extremas como las de Elon Musk, quien ha solicitado reglamentar la inteligencia artificial "antes que sea demasiado tarde", puesto que esta representa una "amenaza contra la humanidad", hay otras que difieren completamente, ya que sostienen que no se debería hacer nada porque el futuro es incierto y crear normas para algo que aún se desconoce cómo va a funcionar, es ineficiente; por tanto, el mismo mercado se encargará de encaminar a la tecnología en el sentido esperado por los seres humanos .

El problema radica en que, en una era de innovación tecnológica constante, compleja y disruptiva, saber qué, cuándo y cómo estructurar las leyes que regulen la tecnología se ha convertido en una tarea sumamente compleja. Los legisladores pueden sentirse presionados al regular una situación sin tener hechos, información y conocimiento suficiente para ello, lo que conllevaría a malas decisiones. Por el contrario, también puede darse el caso que al no tener certeza sobre aquello que se pretende regular, se produzca una "parálisis legislativa" y la realidad colisione contra un ordenamiento jurídico desfasado, afectando con ello la entrada al mercado de nuevas tecnologías.

En ese sentido, la prudencia puede coadyuvar tanto al desarrollo tecnológico como también puede conllevar a un reforzamiento del status quo, promoviendo que las nuevas tecnologías luchen por llegar y consolidarse en el mercado de manera oportuna; mientras que la acción puede traer como consecuencia una sobrerregulación que desincentive la creación y el uso de estas nuevos procesos.

Tomando en consideración la entrada de nuevas tecnologías, desde un punto de vista macro, existe suficiente evidencia como para señalar que el ser humano tiene dos caras y que aquello que se creó para un mejor futuro también puede ser utilizado para uno muy oscuro. En otras palabras, la Tecnología tiene el poder para promover el bien y también el mal. A modo de ejemplo, se crean las impresoras 3D para "democratizar" la Revolución Industrial y que cada persona tenga el poder de crear sus propios diseños; sin embargo, encontramos que estas también sirven para crear armas o para convertir compuestos químicos en drogas caseras.

También podemos citar como ejemplo a la Nanotecnología, campo multidisciplinario que comprende áreas como Biología, Química, Física, Ciencia de Materiales, Ingeniería, entre otras; podría llegar a significar la Revolución Científico-Tecnológica más importante que haya conocido la humanidad, más trascendente aún que el desarrollo del internet. Gracias a ella, será posible aumentar la velocidad de los procesadores de computadoras a límites inimaginables, eliminar contaminantes del agua, la tierra o el aire, detectar y destruir rápida y selectivamente las células cancerosas o crear materiales inteligentes, sólo por mencionar algunas de sus aplicaciones más promisorias. A la fecha, ha mostrado avances en el tratamiento de diversas enfermedades, tales como cáncer, enfermedades neurodegenerativas, autoinmunes y cardiovasculares. No obstante, esta también puede ser utilizada para la producción de armamento bélico o espionaje, ya que la fabricación de armas supone la creación de partículas de menor tamaño que los insectos más pequeños.

El progreso tecnológico convive con un lado oscuro donde las buenas ideas e intenciones producen resultados indeseables —las des-

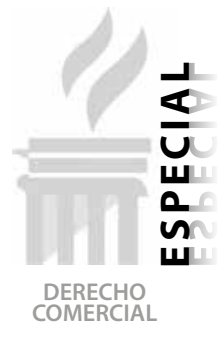


ventajas extremas incluyen armas atómicas y biológicas-. Los muchos y diversos resultados inesperados de la tecnología incluyen situaciones que ponen en riesgo a la humanidad, tales como problemas en el desarrollo operacional de las empresas, puesto que la legislación no tiene una respuesta clara al respecto. Así, por ejemplo, una sociedad que desee aceptar como medio de pago a las criptomonedas, se enfrenta a varias incógnitas, por ejemplo: ¿Es legal en el Perú aceptar estas como medio de pago? ¿Qué sucede si son aceptadas, crecen de valor y sirven para luego adquirir un producto más caro? ¿Se tiene que declarar esto como una ganancia de capital? ¿Qué sucede si no se efectúa? ¿Qué ocurre si la criptomoneda que se ha recibido previamente sirvió para financiar un acto terrorista o para lavar activos?

Por otro lado, si la misma sociedad desea realizar un aumento de capital aportando "bitcoins", el registrador podría verse en aprietos: ¿Es legal? ¿Lo permite el ordenamiento jurídico? ¿Es un aporte dinerario o uno no dinerario? ¿El Bitcoin es dinero? ¿Tiene curso legal? ¿Es dinero electrónico conforme a lo señalado en la Ley que regula las características básicas del dinero electrónico como instrumento de inclusión financiera -en adelante, "Ley 29985"—?

Si bien es cierto que todas estas incógnitas pueden ser resueltas a través de la hermenéutica, lo ideal es crear leyes que faciliten esa tarea y que las personas no pierdan el tiempo en este tipo de interrogantes que podrían ser resueltas de una manera sencilla con una norma. Considero que es importante crear leyes que faciliten la entrada al mercado de estas nuevas tecnologías y se fijen responsabilidades objetivas para aqueIlos que pretenden utilizarlas. Resulta peligroso para su tratamiento aplicar el principio que señala que "lo que no está expresamente prohibido, se encuentra permitido". Asimismo, más riesgoso resulta aún ver como distintos usuarios interpretan a su conveniencia las normas existentes.

\section{INTELIGENCIA ARTIFICIAL}

Tal como señalamos en la introducción del presente artículo, "Vital" marcó un hito en la estructura societaria, puesto que es considerada la primera Inteligencia Artificial que se desarrolló en el proceso de toma de decisiones en el mundo corporativo. Esto significa que la Inteligencia Artificial también puede jugar un papel importante en el gobierno corporativo, dado que los procesos relacionados a la toma de decisiones pueden ser simplificados, transformando las grandes decisiones en un proceso basado en datos experimentales, prediciendo mejor el resultado futuro de tales decisiones.

La Inteligencia Artificial es definida por el diccionario Oxford English Dictionary como el uso de computadoras para tareas que normalmente necesitan de la inteligencia humana' ${ }^{\prime}$. La primera definición fue esbozada por John McCarthy, profesor de Darthmouth College en 1956 al señalar que:

\section{“(...) la ciencia y la ingeniería de crear máqui- nas inteligentes, especialmente programas de computación inteligentes, está relacionada con la tarea similar de utilizar ordenadores para comprender la inteligencia humana, pero la IA no se limita a métodos que sean observables biológicamente".}

Por su parte, Margaret Rouse ${ }^{2}$ señala que es la simulación de procesos de inteligencia humana realizadas por máquinas, especialmente sistemas informáticos. Estos procesos incluyen el aprendizaje —la adquisición de información y las reglas para usar la información-, el razonamiento - el uso de las reglas para llegar a conclusiones aproximadas o definitivas - y la autocorrección. Las aplicaciones particulares de la Inteligencia Artificial incluyen sistemas expertos, reconocimiento de voz y visión artificial. En mi opinión, la Inteligencia Artificial

1. Oxford English Dictionary. Disponible en: $<$ https://en.oxforddictionaries.com/ definition/artificial intelligence $>$.

2. ROUSE, Margaret. Al (Artificial Intelligence). Disponible en: <//searchenterpriseai.techtarget.com/definition/AlArtificial-Intelligence>. 
es la capacidad de una máquina de imitar el comportamiento humano.

Cabe señalar que la Inteligencia Artificial se segmenta en "Strong Al" —Inteligencia Artificial Fuerte- y "Weak Al" —Inteligencia Artificial Débil. La primera se da cuando una máquina es capaz de equipar o superar la inteligencia humana $y$, por lo tanto, puede realizar tareas comúnmente asociadas al ser humano de manera excelente - por ejemplo C-3PO de Star Wars-; mientras que la segunda se configura cuando una máquina sólo puede recrear algunos elementos de la inteligencia humana - este es el caso de Siri, el asistente personal de Apple-

En este contexto, las normas legales deberán adaptarse a esta realidad, puesto que la entrada de esta nueva tecnología abarca una variedad de situaciones y relaciones que se verán afectadas. A manera de ejemplo, en medicina, existen programas como "Da Vinci" y el "CyberKnife" que son equipos de cirugía robótica; es decir, un robot es quien realiza la labor del médico. Google, por su parte, viene desarrollando el proyecto "Waymo", el cual tiene la finalidad de crear automóviles que puedan conducirse de forma autónoma, detectando otros vehículos, señales de tráfico, peatones, etc. En Estados Unidos, una firma de abogados ha contratado a "Ross", un sistema de Inteligencia Artificial creado por IBM, el cual analiza la legislación existente respecto a una determinada pregunta y brinda una respuesta estructurada, reemplazando así el trabajo de un abogado.

Por consiguiente, son distintos los campos en los cuales se desarrolla esta nueva tecnología $y$, por tanto, son diversas las ramas del Derecho que tendrán interacción con la inteligencia artificial. Algunos campos que tendrán que encontrar nuevas soluciones a los problemas que la realidad presente son la Responsabilidad Civil, Derecho Contractual, Propiedad Intelectual, Derecho Tributario, Derecho Administrativo, entre otros. En este orden de ideas, encontramos al Derecho Societario, el cual no estará exento del cambio que implicará el uso de estos sistemas en las corporaciones y la empresa.
Imagine que en un futuro no muy lejano designar a una Inteligencia Artificial como miembro del directorio sea considerada como algo cotidiano en la realidad corporativa en el Perú. Esto presentaría muchos problemas a nivel práctico, ya que los legisladores no visualizaron este supuesto al momento de crear la Ley General de Sociedades - en adelante, "LGS" - . En ese sentido, si una empresa peruana decidiera innovar e invertir varios millones de dólares para que un algoritmo como "Vital" tome las decisiones empresariales pertinentes, actualmente no podrían por las razones que detallo a continuación.

En primer término, se tendría que modificar el artículo 160 de la LGS, puesto que dispone que el cargo de director recaiga solo en personas naturales. Esta tendría que permitir que los programas de software que contengan inteligencia artificial también puedan ser nombrados como directores de empresa. Ahora bien, ¿por qué modificar la LGS? ¿por qué permitir que un programa informático pueda tener la calidad de director? ¿por qué no utilizar al programa y que las personas naturales que conformen el directorio tomen las decisiones empresariales correspondientes en base a la información proporcionada por este? Todas estas interrogantes son válidas; sin embargo, el Derecho Corporativo Transaccional debe fomentar la libertad de empresa. Si un empresario quisiera designar a una Inteligencia Artificial como miembro del directorio, ya sea porque no confía en los humanos o porque quiere promocionarse como empresa innovadora o porque cree que es el camino correcto para poder llevar a cabo su emprendimiento, debería poder hacerlo y la legislación societaria tendría que promover dicha opción a fin de incentivar la innovación y creatividad dentro del campo empresarial.

En el supuesto que se modifique este artículo y un miembro del directorio pueda ser un"softwa$r e^{\prime \prime}$, cabe preguntarse si al menos una persona natural debería conformar parte de este órgano societario. ¿Qué sucedería si todos los miembros del directorio no son humanos? ¿A quién se le asignaría la responsabilidad señalada en el artículo 177 de la LGS? ¿Qué ocurriría si la inteligencia artificial causa algún tipo de daños y per-

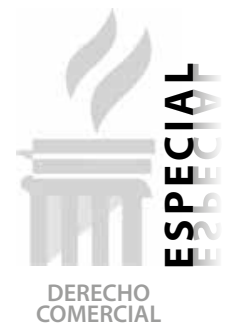


juicios a la sociedad, accionistas o terceros por acuerdos o actos contrarios a la ley o al estatuto? ¿Cómo podrían responder de forma ilimitada y solidaria? La asignación de responsabilidad a la Inteligencia Artificial es un tema bastante complejo, debido a que se tendría que imputar responsabilidad a un director o a un gerente o a un accionista por aquello que la Inteligencia Artificial decidió. Cabe recordar que el desarrollo actual de estos sistemas se basa en los procesos de aprendizaje profundo - machine learning-. Es decir, están diseñados para aprender por sí mismos y de forma automática, siendo capaces de identificar patrones complejos entre millones de datos y elaborar un conjunto de fórmulas y comandos que constituyen las reglas que les permiten descubrir y descifrar cosas por su cuenta; pero, el ser humano puede desconocer aquello que la Inteligencia Artificial está aprendiendo y realizando.

Otro problema - no menor- es que, al igual que los humanos, la Inteligencia Artificial podría aprender a hacer trampa e inclusive a cometer actos ilegales. En el mundo de la tecnología, existe lo que se denomina "black box", donde el ser humano no es capaz de entender el funcionamiento interno de la inteligencia artificial. Esto se puede deber a un comportamiento "doloso" por parte del sistema o simplemente que la Inteligencia Artificial produzca de pronto resultados erróneos, fuera del sentido común. En el 2017, Facebook tuvo que apagar una Inteligencia Artificial que había creado su propio lenguaje. Hace poco, el Massachusetts Institute of Technology —en adelante, "MIT" — creó a Norman - por Norman Bates, personaje de la película "Psycho" de 1961- quien es considerado como la primera inteligencia artificial psicópata tras haber visto los resultados del Test de Rorschach, dicho programa presenta trastornos mentales. Estos ejemplos nos prueban que esta tecnología puede virar hacia una dirección que no necesariamente esté alineada con la de su titular. ¿Qué sucedería si el software tiene la capacidad de adquirir información privilegiada en el mercado de valores y en base a ello toma las decisiones en el directorio? ¿En estos supuestos a quién se hace responsable? ¿A la persona jurídica? ¿A los accionistas? ¿A los demás directores? ¿Al Gerente General?

Es indudable que, a medida que se comience a utilizar esta tecnología en las empresas, la práctica nos colocará en situaciones imprevisibles y que a la fecha no existe una fórmula que ayude a prever todas esas situaciones y solucionarlas de manera eficiente. No por ello se debería prohibir el uso de estos sistemas. Lo que propondría es que, en un inicio, aquellas empresas que quieran designar a programas de Inteligencia Artificial como directores, deberán pagar un impuesto, cuya recaudación debería ser utilizada para crear un fondo común que sirva para pagar multas e indemnizaciones. De esta forma, la norma sería preventiva y no reactiva. Asimismo, lo más sencillo, en una primera instancia, sería que la composición del directorio estuviese conformado en su mayoría por personas y que el cargo de presidente del directorio también recayera en una persona.

Por otro lado, se estima que, en los próximos años, la Inteligencia Artificial comience a reemplazar numerosos puestos de trabajo, inclusive los cargos gerenciales. La investigación de Accenture "Judgment calls: Preparing leaders to thrive in the age of Intelligent Machines" ${ }^{\prime \prime}$, demuestra que un punto a cambiar en el futuro, con la llegada de nuevas aplicaciones y programas de Inteligencia Artificial, es que se debe disminuir en un $25 \%$ el tiempo destinado a coordinación y cambiar las prioridades a temas de estrategia, innovación, colaboración con su equipo y solución de problemas que, a su vez, involucra nuevas destrezas relacionadas a la inteligencia emocional, habilidades interpersonales y toma de decisiones. La realidad nos demuestra que al igual que "Vital", el conglomerado japonés Hitachi, implementó el primer"gerente robótico"en una de sus bodegas,

3. SHANKS, Ryan; SINHA, Sunit y THOMAS, Robert. "Preparing leaders to thrive in the age of intelligent machines". Accenture Strategy, 2016. Disponible en: <https://www.accenture.com/t20170411T174032Z w /us-en/ acnmedia/PDF-19/Accenture-Strategy-Workforce-Judgment-Calls-V2.pdf >. 
a fines de 2015. Esto demuestra que, en un futuro no muy lejano, nos tendremos que enfrentar ante esta nueva realidad.

Ahora bien, las mismas interrogantes surgen en el caso de que se designe como gerente a una máquina con Inteligencia Artificial. ¿Cómo se le hace responsable a una Inteligencia Artificial que tiene el cargo de Gerente General? ¿Cómo se le pueden otorgar poderes para que realice actos de administración? ¿Podría figurar en una partida electrónica que cierta tecnología tiene poderes para cierto tipo de actos? Creería que, para llegar a ese punto, el sistema peruano debería ser "pro-Inteligencia Artificial", es decir, que el mercado nacional se encuentre en la capacidad de permitir el tráfico mercantil a través de este sistema. Por ejemplo, que el sistema financiero pueda identificar a aquellas empresas que han designado como gerente a un programa y que estos a su vez tengan un software que pueda determinar los tipos de actos que la Inteligencia Artificial pueda realizar. Al igual que los bancos, los organismos públicos también deberían tener la tecnología necesaria para poder facilitar este tipo de transacciones. Por ejemplo, que la Inteligencia Artificial pueda modificar formularios a través de SUNAT ONLINE o que la plataforma de SUNARP integre un espacio para que las distintas inteligencias artificiales de diversas empresas puedan realizar ciertos trámites. En otras palabras, mientras que el Estado peruano no haya impulsado un gobierno digital y el mismo mercado peruano no se encuentre a la vanguardia, la actuación de cualquier "gerente robótico" debería limitarse, ya que de lo contrario a nivel práctico las sociedades se enfrentarían a un sinnúmero de problemas en aquellos actos de administración que se necesiten ejecutar en su quehacer diario.

Si bien uno podría pensar que lo escrito hasta el momento es parte de la ciencia ficción, cabe recordar que a la fecha los "softbots" o robots bursátiles son comúnmente usados para realizar transacciones financieras: Entre el $80 \%$ y $85 \%$ de la actividad bursátil en los Estados Unidos y Gran Bretaña es llevada a cabo mediante flash traders - corredores de alta frecuencia- que pueden colocar entre diez mil y veinte mil órdenes por segundo e inclusive en el 2010 provocó un fallo histórico cuando la Bolsa de Nueva York se desplomó casi 1.000 puntos en apenas media hora por un algoritmo mal programado que acarreó pérdidas de miles de millones de dólares.

En ese sentido, tal como dijo Charles Darwin:"No es la más fuerte de las especies la que sobrevive y tampoco la más inteligente. Sobrevive aquella que más se adapta al cambio". En ese orden de ideas, creer que la Inteligencia Artificial no afectará la estructura societaria en las empresas y, por consiguiente, el tráfico mercantil es justamente resistirse al cambio. Anticiparse a estos sucesos permitirá a miles de empresarios adaptarse de forma rápida a esta nueva era. Resulta peligroso desconocer los posibles escenarios que se pueden presentar en la práctica debido a la utilización de esta nueva tecnología.

\section{BLOCKCHAIN}

En el año 2009, Satoshi Nakamoto creó el Bitcoin, una criptodivisa que tiene como "back end" al "Blockchain" o "cadena de bloques", que es una base datos, o un libro de contabilidad público mundial distribuido en una red descentralizada, en el que se anotan todo tipo de operaciones utilizando la criptografía 4 . En otras palabras, el "Blockchain" funciona igual que un libro mayor de contabilidad, pero, en este caso, los apuntes son públicos y descentralizados. Asimismo, esta cadena de bloques está diseñada exclusivamente para evitar su alteración una vez que los datos han sido publicados ${ }^{5}$.

4. CARRASCOSA, Cristina. "¿Qué es el blockchain y por qué va a cambiar (casi) todo?" Disponible en: <http://ecija.com/ blockchain-va-cambiar-todo/>.

5. A modo de ejemplo, supongamos que una nave alienígena aterrice en plena Plaza de Armas de Lima y de allí salen dos extraterrestres que saludan al público terrestre para luego regresarse a su planeta de origen. Una vez 
Dan Tapscott, escritor del libro denominado "La Revolución del Blockchain" la define como:

"Un libro mayor o una gran base de datos dis-
tribuida globalmente, que opera en millones
de dispositivos y se encuentra abierta a cual-
quier persona, donde no sólo la información
es almacenada y gestionada de forma segura
y privada, sino cualquier cosa de valor como
el dinero, los títulos, los actos, las identidades,
incluso los votos podrán ser administradas de
esta manera. La confianza se establece a tra-
vés de la colaboración masiva y criptografía
inteligente en lugar de utilizar intermediarios
poderosos como los gobiernos y los bancos".

Estrictamente, el término "Blockchain" hace referencia al libro registro -ledger - inmutable que contiene la historia completa de todas las transacciones que se han ejecutado en la red con una marca temporal. Así pues, cualquier persona, en cualquier momento, puede acceder a consultar ese registro teniendo la certeza que su información es actualizada y consistente con el resto de nodos de la red, a pesar de que se trate de una red descentralizada ${ }^{7}$.

Imaginemos una hoja Excel o un fichero de texto con dos columnas, en donde una tenga un identificador con las siguientes letras, "abc" $y$, en la otra, un número, por ejemplo, "20". Es decir, "abc" se identifica con el número "20". Ahora, supongamos que esa hoja Excel se encuentra distribuida en miles de ordenadores, sincronizándose estos de manera frecuente. Aunque uno de estos desapareciera, no afectaría a la red, dado que los códigos "abc" $y$ " 20 " se encuentran almacenados de forma descentralizada. Por tanto, si se altera algún ordenador, los demás demostrarían que dicho registro fue alterado.

Ahora bien, cabe preguntarse ¿quién controla que esos apuntes sean verídicos y correctos? Lo hacen unos vigilantes voluntarios del "Blockchain", quienes a cambio de una remuneración económica — pagada en "tokens" - comprueban la veracidad de las operaciones que ocurran a través del "Blockchain". Por ejemplo, en una compraventa de un cuadro realizada a través del "Blockchain", los vigilantes voluntarios verifican que: (i) esa misma pieza de arte no haya sido vendida a otro usuario con anterioridad y (ii) la pieza de arte pertenece al vendedor.

Todo ello puede realizarse dentro de lo que se denomina una "prueba de trabajo" - "Proof of Work" - por un nodo, que es básicamente un individuo conectado a un ordenador con capacidad suficiente para procesar los problemas matemáticos a los que va intrínsecamente unido el "bloque" de información que trata de anclar al resto de la cadena y; por los que recibe esa gratificación mencionada anteriormente una vez resueltos estos ${ }^{8}$. En ese sentido, aquellos nodos, los cuales se encuentran separados físicamente los unos de los otros, trabajan de forma consensuada para crear los bloques, y actúan para vigilar y preservar la validez de la cadena. Así, se asegura que la alteración en el "Blockchain" en uno de los ordenadores no altere el "Blockchain" de otro, puesto que, para quedar validado en el "Blockchain", la alteración debería de hacerse en cada copia de cada nodo, lo que es prácticamente imposible.

sucedido este impresionante acontecimiento, se coloca un detector de mentira al $100 \%$ a personas que fueron testigos de dicho suceso y se registra aquello que han visto. Todos cuentan la misma historia, con los mismos detalles, despejando así las dudas y comprobando, sin margen de error, que este evento sucedió. El "Blockchain" pretende ser este "detector de mentiras" que compruebe la autenticidad de los hechos a través de diferentes "nodos" que cumplen el rol de estos testigos. Esta tecnología es una forma de verificación y validación de la información, amparándose en el principio de veracidad de la información, empleando la criptografía.

6. TAPSCOTT, Don. "Blockchain Revolution: How the Technology Behind Bitcoin Is Changing Money, Business and the World". Penguin Random House, 2016.

7. FERNÁNDEZ ESPINOZA, Luz. "Bitcoin y Blockchain: retos y oportunidades para el sector financiero". BBVA, 2016. Disponible en: <https://www.bbva.com/es/bitcoin-blockchain-retos-oportunidades-sector-financiero/>.

8. CARRASCOSA, Cristina. Op cit. 
Como mencionamos anteriormente, el "Blockchain" funciona como soporte del Bitcoin. Sin embargo, esta tecnología revolucionará distintas industrias. A modo de ejemplo, el Banco de Pagos Internacionales publicó un estudio sobre el impacto del "Blockchain" $y$ "ledgers" distribuidos en la industria financiera, concluyendo que es susceptible de reducir el rol de intermediarios de bancos, cámaras de compensación y bancos centrales. Asimismo, en el sector salud, la utilización de firmas digitales en datos basados en "Blockchain", puede controlar la disponibilidad de historias médicas y así mantener su privacidad.

La posibilidad de almacenar datos personales e industriales en una base de datos segura y completa lleva a cualquiera a imaginar todas las implicaciones que ese tipo de tecnología podría tener sobre el futuro de los negocios en muchos sectores. Para demostrar la magnitud del impacto que tendrá el "Blockchain" a nivel societario, podemos señalar que, en julio del 2018, la Delaware General Corporation Law se modificó permitiendo a las compañías mantener sus registros corporativos utilizando esta tecnología. En este Estado se conglomeran más de la mitad de las compañías que listan en bolsa y un $60 \%$ de las compañías que figuran en el ranking Fortune 500 fueron constituidas allí.

Una de las razones por la cual se modificó esta norma es que algunos ven el uso del "Blockchain" como una solución a los problemas que se derivan de mantener actualizados los libros matrícula de acciones y registrar los votos de los accionistas. Asimismo, otra de las razones es que emitir y negociar acciones a través del "Blockchain" crea numerosos beneficios y reduce ciertos costos relacionados con una mayor transparencia de la propiedad, así como una ejecución y liquidación de operaciones más rápida y más barata. Una mejor transparencia impactaría significativamente las oportunidades de ganancias disponibles para los gerentes, inversionistas institucionales y activistas de ac- cionistas, entre otros, porque los incentivos para adquirir propiedad y liquidarla podrían cambiar marcadamente si sus transacciones se pueden observar en tiempo real ${ }^{9}$.

En el Perú, por ejemplo, los libros matrículas de acciones podrían utilizar esta tecnología a fin de evitar todos los problemas derivados con la titularidad de quien aparece allí. Cabe recordar que el artículo 91 de la LGS considera propietario de la acción a quien aparezca como tal en la matrícula de acciones, y que si fuera el caso que se esté litigando la titularidad de las acciones, se admitirá el ejercicio de los derechos de accionista por quien aparezca registrado en la sociedad como propietario de ellas, salvo mandato judicial en contrario. En este libro se inscriben los siguientes actos, de conformidad con el artículo 92 de la mencionada ley:

a) La creación de acciones cuando corresponda, anotándose en un asiento distinto del de emisión en la medida que se trate de actos separados en el tiempo.

b) La emisión de acciones sea que estén representadas por certificados provisionales o definitivos.

c) La transferencia, canjes y desdoblamientos de acciones.

d) La constitución de derechos y gravámenes sobre las mismas.

e) Las limitaciones a las transferencias de acciones.

f) Los convenios entre accionistas o de accionistas con terceros que versen sobre las acciones o que tengan por objeto el ejercicio de los derechos inherentes a ellas.

En ese sentido, así como se permite llevar el Libro de Matrícula de Acciones en un libro especialmente abierto a dicho efecto, $u$ hojas sueltas, ambos de-

9. YERMACK, David. "Corporate Governance and Blockchains". Review of Finance, Vol. XXI, Primera Edición, Enero 2017, pp 7-31. Disponible en: <https://doi.org/10.1093/rof/rfw074>. 
bidamente legalizados ante notario o mediante anotaciones en cuenta, también se podría incorporar al "Blockchain" como una manera de registrar todos los actos mencionados en el artículo 92 de la LGS. De esta forma, se podría contribuir con la transparencia y la celeridad en la anotación en el Libro de Matrícula, dándoles así mayor seguridad a los titulares de las acciones.

Otro de los usos que se podría dar al "Blockchain" en temas societarios es introducir esta tecnología para poder llevar a cabo las Juntas Generales de Accionistas. Al respecto, Anna Lafarre y Cristoph Van der Elst ${ }^{10}$ señalan que en un "Blockchain" privado, administrado y gestionado por la compañía, solo accesible para los accionistas, por ejemplo, el Hyperledger de IBM, la sociedad y los accionistas que poseen acciones suficientes pueden presentar propuestas en las juntas generales de accionistas. Se pueden crear "Smart Contracts" a fin de que se permita estructurar el Libro de Junta General de Accionistas de manera que se tenga en cuenta toda la información relevante, incluidas las reglas de la mayoría y los derechos de acceso que figuran tanto en los estatutos como en la ley. Una vez que se coloca una determinada propuesta en el "Blockchain", los accionistas que poseen acciones de la sociedad son notificados inmediatamente y pueden ejercer sus derechos de voto durante un corto período. Los resultados de la votación pueden estar disponibles instantáneamente, después de un punto de corte, y los requisitos de la mayoría, necesarios para que la decisión sea vinculante y verificable, deben alcanzarse en un período de tiempo específico. Los accionistas pueden verificar sus propias transacciones, pero ninguno de los accionistas debe poder determinar qué decisión de voto tomaron otros accionistas.

Por tanto, con esta tecnología, los accionistas pueden reducir sustancialmente los costos de transacción para la toma de decisiones. Asimismo, también puede ofrecer oportunidades para aumentar la celeridad en este tipo de actos societarios, convirtiendo a la junta en un órgano corporativo rápido y eficiente. Los principales problemas con el actual sistema de votación remota tienen que ver con la transparencia, la verificación y la identificación, cuestiones que están directamente relacionadas con las ventajas de la tecnología "Blockchain".

Por otro lado, un estudio realizado por Holden, Jacobsen y Subrahmanyam en el $2013^{11}$, señala que el "Blockchain" ofrece la posibilidad de mejoras significativas en la liquidez del mercado de valores, ya sea que se utilicen como plataforma principal para el registro e intercambio de acciones o, alternativamente, si los introducen los mercados bursátiles de una manera más limitada para agilizar el proceso de compensación y liquidación posterior a la negociación. Las operaciones bursátiles en Estados Unidos, generalmente, demora tres días hábiles para que ocurra la liquidación total y que la propiedad se traslade formalmente del vendedor al comprador. Durante este intervalo, los fondos pasan entre los corredores y sus clientes, y las acciones se transfieren en los libros de la correduría y el libro mayor de la corporación, todo bajo la supervisión de Depository Trust Clearing Corp. Muchas personas están involucradas en este proceso. En contraste, una venta de acciones utilizando el "Blockchain" podría resolverse de forma más rápida, pues no necesitaría numerosos intermediarios, lo que reduciría los costos que ahora aparecen de diversas formas en las comisiones y los diferenciales de oferta y demanda. Si bien es probable que los mercados bursátiles continúen operando de alguna forma para facilitar la reunión de compradores y vendedores, la liquidez podría aumentar en gran medida en respuesta al menor costo y la mayor velocidad de liquidación.

10. LAFARRE, Anne, VAN DER ELST, Christoph (27 de marzo de 2018). Blockchain Technology for Corporate Governance and Shareholder Activism. European Corporate Governance Institute -ECGI-. Law Working Paper N ${ }^{\circ}$ 390/2018. Tilburg Law School Research Paper No 2018-7. Disponible en: <https://corpgov.law.harvard.edu/2018/03/27/ blockchain-technology-for-corporate-governance-and-shareholder-activism/>.

11. YERMACK, David. Op. Cit. 
Utilizando la misma lógica, en el mercado de valores peruanos también participan numerosos actores, teniendo a distintos intermediaros en el proceso de adquisición o venta de acciones. Esta nueva tecnología podría reducir la injerencia de los mismos y acelerar el proceso de transferencia de estos valores mobiliarios. Es innegable que la tecnología "Blockchain" en un futuro cercano será una pieza clave para registrar los actos societarios que se toman en el devenir de una empresa.

\section{CRIPTOMONEDAS}

Las criptomonedas también afectarán al Derecho Societario y al Comercio Transaccional en general, ya que se han convertido en una de las grandes tendencias del mundo digital. Las monedas virtuales están de moda $y$, aunque todos pensemos en "Bitcoin", hay muchas más criptodivisas que cada vez están ganando más importancia en lo que será el futuro de la economía digital, como Etherum o Ripple.

Una criptomoneda es una moneda virtual que sirve para intercambiar bienes y servicios a través de un sistema de transacciones electrónicas, incorporando los principios de la criptografía para implementar un comercio transparente, anónimo y descentralizado. En otras palabras, son monedas virtuales y tienen las siguientes características:

a) Descentralizadas: Las criptomonedas no se encuentran controladas por ninguna autoridad, banco o institución financiera.

b) Internacionales: Pueden utilizarse por igual en cualquier país del mundo.

c) Voluntarias: La adopción y el uso de estas monedas son voluntarias, ya que, al no estar respaldado por un banco central, nadie se encuentra obligado a aceptarlas como medio de pago. d) Transparentes: Todas las operaciones que se producen en el sistema son registrados en el "Blockchain", garantizando así la transparencia en las transacciones comerciales.

e) Rápidas: Una transacción debería demorar lo mismo así se haga en cualquier parte del mundo, a diferencia del sistema financiero tradicional que, por lo general, una transferencia de dinero puede demorar dependiendo de las circunstancias.

Ahora bien, cabe preguntarse cuál es la naturaleza jurídica de las criptomonedas. ¿Es dinero? ¿Es un bien mueble? ¿Es dinero electrónico? Para ello debemos de definir y explicar qué es el dinero. En un primer momento, el dinero se creó para reemplazar al trueque, ya que facilita el intercambio. El dinero constituye un medio de pago que es aceptado por un determinado grupo de personas y existe una mejor asignación del valor de cada bien que se desea intercambiar. Por tanto, es un instrumento que permite reducir los costos de transacción. En la actualidad, el dinero comúnmente se compone de monedas y billetes, las cuales poseen las siguientes características $^{12}$ :

a) Portabilidad: El dinero debe ser fácilmente transportable.

b) Durabilidad: El dinero que no sea durable pierde su valor como moneda - los soldados romanos recibían su pago en sal, por ello se habla de salario-

c) Divisibilidad: El dinero debe ser fácilmente divisible en partes iguales para permitir la compra de unidades más pequeñas.

d) Uniformidad: Para ser útil, el dinero debe ser estandarizado. Sus unidades deben ser de igual calidad y sin que existan diferencias físicas entre sí.

12. BARCHI VELAOChaGA, Luciano. "Código Civil Comentado", Tomo VI. Editorial Gaceta Jurídica, primera edición, Lima: 2004, p.517. 
e) Reconocimiento: El dinero debe ser fácilmente identificable.

El dinero siempre tendrá estas características; sin embargo, no todo bien que las tenga será considerado dinero, desde una perspectiva jurídica. Esto es debido a que el dinero debe tener curso legal, lo que significa que los billetes y monedas que el Banco Central de Reserva pone en circulación son de aceptación forzosa para el pago de toda obligación, pública o privada. En el Perú, el Sol -S/- es desde el 15 de diciembre de 2015, la unidad monetaria de curso legal y el artículo 4 de la Ley Orgánica del Banco Central de Reserva -en adelante, "BCR" - establece que la emisión de billetes y monedas es facultad exclusiva del Estado, quien la ejerce por intermedio del BCR. Por consiguiente, las criptomonedas no tienen curso legal en el Perú y, por tanto, no podrán ser consideradas como dinero.

Tampoco se podría decir que las criptomonedas son dinero electrónico, puesto que, al no tener curso legal, su aceptación como medio de pago no es obligatoria y, por tanto, no encajaría en la definición establecida en el artículo $2^{13}$ de la Ley 29985 -Ley que regula las características básicas del dinero electrónico como instrumento de inclusión financiera. Asimismo, según el artículo 3 de esta ley, sólo pueden emitir dinero electrónico las empresas que operan bajo el ámbito de supervisión de la Superintendencia de Banco, Seguros y AFP — "SBS" - . En cambio, las criptomonedas pueden ser creadas por cualquiera. Por tanto, es seguro señalar que las criptodivisas no pueden ser catalogadas como dinero electrónico en el Perú.
En mi opinión, las criptomonedas son bienes muebles, inmateriales, de naturaleza digital, fungible y no consumible. Un bien es la cosa que está dentro del patrimonio de una persona y que es susceptible de valoración económica. Bajo esa misma línea, un bien mueble es aquél que puede trasladarse de un lugar a otro y pueden ser materiales o inmateriales. Podemos decir con certeza que una criptomoneda es un bien inmaterial porque al no tener una presencia corpórea no puede ser percibido por los sentidos. Asimismo, es un bien que sólo existe en el mundo digital. Finalmente, es fungible puesto que las criptodivisas pueden ser recíprocamente sustituidas por otras y son no consumibles porque se pueden usar sin que éstas se agoten.

Ahora bien, ¿qué sucedería si un grupo de emprendedores deciden constituir una sociedad con "Bitcoins" o que accionistas en una sociedad anónima decidan realizar un aumento de capital a través de "Etherum"? ¿Se podría en el Perú? Creemos que sí se podrían constituir sociedades o incrementar el capital social aportando criptomonedas, ya que tal como señala PALMADE$\mathrm{RA}^{14}$, legalmente, no existe ninguna limitación o prohibición respecto a las características de los bienes materia de aporte. La concreción del principio de integridad del capital social y la obligación que asumen los socios de contribuir al fin social demanda únicamente que el objeto del aporte tenga un contenido económico y sea idóneo para alcanzar el fin buscado.

Por consiguiente, los fundadores de una Sociedad Anónima podrían constituir una sociedad

13. Artículo 2 de la Ley 29985.- "El dinero electrónico es un valor monetario representado por un crédito exigible a su emisor, el cual tiene las siguientes características:
a) Es almacenado en un soporte electrónico.
b) Es aceptado como medio de pago por entidades o personas distintas del emisor y tiene efecto cancelatorio.
c) Es emitido por un valor igual a los fondos recibidos.
d) Es convertible a dinero en efectivo según el valor monetario del que disponga el titular, al valor nominal.
e) No constituye depósito y no genera intereses".

14. PALMADERA ROMERO, Doris. "Manual de la Ley General de Sociedades". Editorial Gaceta Jurídica. Primera Edición, Lima, Noviembre, 2009, p.78. 
aportando criptomonedas, al igual que los accionistas de este tipo de sociedad podrían llevar a cabo una Junta General de Accionistas, aprobar un aumento de capital, en donde se aporten criptomonedas en calidad de un aporte de bienes no dinerarios, insertar un informe de valorización, de acuerdo al artículo 27 de la LGS y se requerirá la certificación del gerente general de haberlos recibido, conforme al literal f) del artículo 35 del Reglamento de Registro de Sociedades.

Si bien no se necesita de una nueva legislación para poder admitir que un accionista aporte criptomonedas al capital de la sociedad, cabe preguntarse si es que es necesario regularlas en el Perú. No son pocas las personas que creen que se debería regular este mercado, puesto que al no tener un marco legal que las respalde, las personas pueden aprovechar estos vacíos jurídicos para realizar operaciones ilegales.

Dentro de este contexto, hay quienes sostienen que un marco regulatorio no puede aplicarse a las monedas digitales, puesto que dentro de sus características principales están la descentralización y su vocación internacional. Por tanto, las transacciones mediante criptomonedas no están validadas por una entidad central, lo que significa que no hay cargos bancarios o de plataforma asociados con los pagos. Asimismo, dado que no se circunscriben a un territorio, no existen comisiones por pagos que atraviesan las fronteras, y la transacción funciona de la misma manera, sin importar en donde se encuentren los contratantes o la persona con la que se realiza un negocio.

En ese sentido, las criptomonedas permitirán a aquellas personas que no pueden acceder al sistema financiero tradicional a trasladar recursos de un país a otro o incluso de un continente a otro en minutos, sin formularios, sin cuentas bancarias $y$, especialmente, sin intermediaros que cercenan un importante porcentaje de la remesa. Por ejemplo, todas las personas que trabajan en el extranjero y necesitan enviarles sus ingresos a sus familiares, podrán utilizar esta tecnología sin ver que gran porcentaje de estos se ven disminuidos por los gastos relacionados al envío.

Sin embargo, las criptodivisas operan en una zona tan gris que se prestan para distintas actividades ilegales. Por tal motivo, la Unión Europea manifestó su preocupación señalando lo siguiente:

\begin{abstract}
"Las transacciones con monedas virtuales se benefician de un grado de anonimato mayor que las transferencias de fondos financieros clásicas y por tanto conllevan un riesgo de que las monedas virtuales se puedan usar por parte de organizaciones terroristas para ocultar transferencias financieras. Posibles riesgos posteriores tienen que ver con la irreversibilidad de las transacciones, medios de tratar con operaciones fraudulentas, la naturaleza opaca y tecnológicamente compleja de la industria y la falta de salvaguardas regulatorias"15.
\end{abstract}

De igual forma, en Estados Unidos fue presentada el Senate Bill $N^{\circ} 1241$, el 25 de mayo del año 2017, con el fin de combatir el lavado de dinero, el blanqueo de capitales y el financiamiento al terrorismo, y en ella califican a las criptomonedas como métodos de pago de origen cuestionable y de alto riesgo en materia de lavado de activos. Un ejemplo de ello lo encontramos en el caso BTC-e, empresa que manejaba alrededor del $2.5 \%$ de todo el volumen de intercambio de Bitcoin a nivel internacional y que ha sido calificado por el Departamento de Justicia de Estados Unidos como una de las entidades más grandes en el campo de lavado de dinero en el mundo. En ella se podía realizar el comercio entre el dólar estadounidense, y las criptomonedas tales como Bitcoin, Litecoin, Namecoin, Novacoin, Peercoin, Dash y Ethereum. Dichos ingresos pro-

15. EUROPEAN COMISSION (05 de julio de 2016). Proposal for a Directive of the European Parliament and of the Council amending Directive - EU-2015/849 on the prevention of the use of the financial system for the purposes of money laundering or terrorist financing and amending Directive 2009/101/EC. Disponible en: < http://data. consilium.europa.eu/doc/document/ST-10678-2016-INIT/en/pdf>. 
venían de la piratería, la extorsión cibernética y el tráfico de drogas. BTC-e fue utilizado por criminales en todo el mundo.

Al no existir una regulación clara al respecto en el Perú, una sociedad peruana que decidiera aceptar "Bitcoins" u otra criptomoneda como medio de pago podría verse envuelta en un proceso penal, poniendo en riesgo a sus representantes legales, inclusive pudiendo ser sancionados administrativamente si no cuentan con un modelo de prevención de delitos debidamente implementado al amparo de la Ley 30424, ya que el artículo 2 del Decreto Legislativo 1160 -Decreto Legislativo de Lucha Eficaz Contra el Lavado de Activos y Otros Delitos Relacionados a la Minería llegal y Crimen Organizado- establece que:

"El que adquiere, utiliza, guarda, custodia, recibe, oculta o mantiene en su poder dinero, bienes, efectos o ganancias, cuyo origen ilícito conoce o debía presumir, con la finalidad de evitar la identificación de su origen, su incautación o decomiso, será reprimido con pena privativa de la libertad no menor de ocho ni mayor de quince años y con ciento veinte a trescientos cincuenta días multa" (el subrayado es nuestro).

En ese sentido, al no estar reguladas en el mercado y a sabiendas que muchas criptomonedas garantizan el anonimato de sus usuarios al momento de realizar transacciones, no queda claro si es que una sociedad "debía presumir" el origen de esas criptomonedas, lo cual claramente genera un riesgo comercial muy alto para aquellas compañías que decidan aceptarlas como medio de pago.

Por otro lado, las corporaciones a nivel mundial están considerando crear sus propias criptomonedas a fin de utilizarlas como "Tokens" o tipos de representación de valor. Es decir, las criptomonedas pueden ser utilizadas como tokens de utilidad, que son básicamente los que puedes usar dentro de un ecosistema para participar en un servicio o utilidad. Un ejemplo son los casinos, donde uno compra sus propios tokens o fichas que luego se pueden utilizar en sus máquinas y restaurantes. Por ejemplo, la compañía ZeroEdge, ha desarrollado su propia moneda virtual para ser utilizada en su servicio de casino en línea; así, su objetivo y uso es bien definido, claro y único: la utilización en su casino en línea. Por su lado, existen rumores que señalan que Facebook está planeando lanzar su propia criptomoneda, la cual serviría para hacer transacciones dentro de la plataforma, que van desde pagos móviles, hasta envío de divisas a contactos y adquirir productos en su Marketplace, con lo cual esto podría acelerar e incentivar a otras empresas a crear sus propias criptomonedas.

Lo cierto es que en los últimos años las empresas han creado cientos de tokens por la facilidad con la que se pueden desarrollar, y sus dueños se han dado cuenta de que pueden ser utilizados para financiarse. A este proceso de financiamiento se le denomina Initial Coin Offering — en adelante, "ICO" - , que podemos traducir como "Oferta Inicial de Monedas", el cual consiste en que las sociedades crean un token, deciden cuál será su valor o para qué se va a utilizar, y lo ofertan en el mercado. Si bien el nombre se parece bastante al "Initial Public Offering" u Oferta Pública Inicial, el /CO no supone convertirse en accionista de la sociedad.

Cabe cuestionarse qué sucedería si una sociedad peruana decide crear su propia criptomoneda e iniciar una "Oferta Inicial de Monedas". ¿Sería legal o no? Nuevamente, al no contar con un marco legal adecuado, se podría restringir el uso de esta práctica aduciendo que la sociedad que lanza dicha oferta, se encuentra captando dinero del público sin autorización de la Superintendencia de Banca y Seguros, vulnerando así lo dispuesto en el artículo11 de la Ley 26702 —Ley General del Sistema Financiero y del Sistema de Seguros y Orgánica de la Superintendencia de Banca Segu$\operatorname{ros}^{16}$ - En otras palabras, sucedería algo similar a lo que ocurre con el "Crowdfunding", que a la fecha no cuenta con una norma que permita este tipo de

16. Artículo 11 de la Ley N²6702.- Actividades que requieren de autorización de la Superintendencia

Toda persona que opere bajo el marco de la presente ley requiere de autorización previa de la Superintendencia de 
financiamiento, dado que la regulación peruana señala que toda invitación que una persona natural o jurídica dirija al público en general para realizar cualquier acto referido a la colocación o venta de valores mobiliarios es oferta pública de valores, debiendo observar el régimen legal establecido y ser objeto de supervisión de la Superintendencia de Mercado de Valores.

Finalmente, se advierte que las criptomonedas han levantado inquietudes en las autoridades tributarias de diferentes países por la dificultad para rastrear las transacciones $y$, por lo tanto, por la posibilidad que se utilicen para evadir impuestos. Imaginemos que un peruano decide que le paguen en Bitcoins y luego este decide comprar productos en establecimiento comerciales que aceptan al Bitcoin como medio de pago. Lo cierto es que esa persona difícilmente declararía sus ganancias a la administración tributaria - Superintendencia Nacional de Administración Tributaria, en adelante "SUNAT" - . Por otro lado, aquellos que sí quieran tributar sus ganancias a través de las criptodivisas, se enfrentarían a problemas prácticos como, por ejemplo, saber qué tipo de formulario se debe utilizar para declarar.

\section{CONCLUSIÓN}

Henry Ford alguna vez dijo que "el verdadero progreso es el que pone la tecnología al alcance de todos". Sin embargo, este no podrá ser beneficioso si es que no existen las normas legales correspondientes para que todos los actores en el mercado puedan aprovecharla. El desarrollo comercial debe de ir de la mano con una legislación flexible que se adapte a la realidad. Las nuevas tecnologías presentan retos en la aplicación del Derecho y si queremos crecer como sociedad y promover el desarrollo empresarial debemos enfocarnos en darle un marco claro, sencillo y práctico a los emprendedores a fin de facilitar su actividad. Los legisladores se enfrentan a un desafío bastante grande pues las normas que regulen estos fenómenos no deben sobrepasarse y limitar el progreso, ni tampoco quedarse cortas y permitir la invasión de derechos.

Las nuevas tecnologías como la inteligencia artificial, el "Blockchain"y las criptomonedas tienen el potencial de cambiar el mundo empresarial tal como lo conocemos y el Derecho Comercial debe funcionar como un catalizador en la creación y uso de estos nuevos conocimientos que pueden ser muy beneficiosos para la población. En ese sentido, cualquier reforma en este campo del Derecho que tenga por finalidad incluir el uso de estas tecnologías debe asegurarse que dichas regulaciones tomen en consideración los impactos económicos, sociales y técnicos. En otras palabras, este proceso debe tener en cuenta los efectos que la regulación puede tener respecto a la innovación.

En esta era de cambios tecnológicos, la velocidad es la clave del éxito respecto a las actividades comerciales. Las regulaciones burocráticas e ineficientes no pueden ser toleradas por el empresario peruano. Los cambios normativos no pueden implicar una carga administrativa pesada que afecte de forma negativa a los microempresarios, puesto que esto implica que los empresarios dedican mucho tiempo a trámites lentos en vez de enfocarse en innovar y a poner en marcha su idea de negocio utilizando estas nuevas tecnologías.

A modo de conclusión, es innegable que las nuevas tecnologías afectarán necesariamente al Derecho Comercial, ya que esta rama debe de mantenerse a la vanguardia de las nuevas realidades que se presentan en el tráfico mercantil y en el impacto que esto puede tener en los distintos actores en el mercado. Ahora bien, la pregunta que debemos de formularnos es: ¿Cómo queremos que la tecnología incida en el Derecho Comercial?

acuerdo con las normas establecidas en la presente ley. En consecuencia, aquella que carezca de esta autorización, se encuentra prohibida de:

1. Dedicarse al giro propio de las empresas del sistema financiero, y en especial, a captar o recibir en forma habitual dinero de terceros, en depósito, mutuo o cualquier otra habilitación de fondos, bajo cualquier modalidad contractual. $(\ldots)^{\prime \prime}$ 